CRYSTALLOGRAPHIC COMMUNICATIONS

ISSN 2056-9890

Received 25 August 2017

Accepted 27 September 2017

Edited by W. T. A. Harrison, University of Aberdeen, Scotland

Keywords: crystal structure; 3-alkylidene-3Hisobenzofuran-1-ones; delocalization; enolate.

CCDC reference: 1543053

Supporting information: this article has supporting information at journals.iucr.org/e

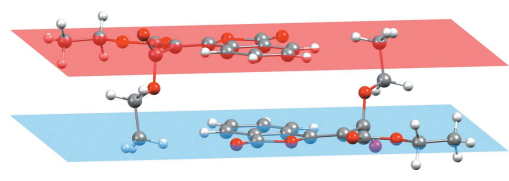

OPEN $\odot$ ACCESS

\section{Crystal structure and identification of resonance forms of diethyl 2-(3-oxoiso-1,3-dihydrobenzo- furan-1-ylidene)malonate}

\author{
Mikhail S. Tyumentsev, ${ }^{\mathrm{a} *}$ Mark R. StJ. Foreman, ${ }^{\mathrm{a}}$ Britt-Marie Steenari ${ }^{\mathrm{a}}$ and \\ Alexandra M. Z. Slawin ${ }^{b}$ \\ ${ }^{a}$ Nuclear Chemistry and Industrial Materials Recycling, Department of Chemistry and Chemical Engineering, Chalmers \\ University of Technology, Gothenburg, Sweden, SE 41296, and ${ }^{\mathbf{b}}$ School of Chemistry, University of St. Andrews, Purdie \\ Building, North Haugh, St. Andrews, Fife, Scotland, KY16 9ST. *Correspondence e-mail: miktyu@chalmers.se
}

The reaction of diethyl malonate with phthaloyl chloride in acetonitrile in the presence of triethylamine and magnesium chloride results in the formation of the title compound, diethyl 2-(3-oxo-1,3-dihydro-2-benzofuran-1-ylidene)propanedioate, $\mathrm{C}_{15} \mathrm{H}_{14} \mathrm{O}_{6}$. One of the ester groups of the diethyl malonate fragment is almost coplanar with the isobenzofuran unit, while the plane of the other group is perpendicular to it [dihedral angles $=5.45(3)$ and $83.30(3)^{\circ}$, respectively]. The $\mathrm{C}-\mathrm{C}$ and $\mathrm{C}-\mathrm{O}$ distances both in the heterocyclic furan ring and the diethyl malonate fragment are indicative of the dipolar delocalization occurring within the isobenzofuran unit. This delocalization is likely to be responsible for the unusual intermolecular $\mathrm{O} \cdots \mathrm{O}$ contact [2.756(2) $\AA$ ], established between the $\mathrm{O}$ atom of the furan ring and the carbonyl $\mathrm{O}$ atom of the diethyl malonate fragment. In the crystal, weak $\mathrm{C}-\mathrm{H} \cdots \mathrm{O}$ interactions are observed, which link the molecules into [100] chains.

\section{Chemical context}

The structural analysis of diethyl 2-(3-oxoisobenzofuran$1(3 H)$-ylidene)malonate (I) was undertaken as part of a study into the synthesis of new reagents for the recovery of trivalent lanthanide metal ions by liquid-liquid extraction. We intended to prepare 2,2'-phthaloylbis $\left(N, N, N^{\prime}, N^{\prime}\right.$-tetrabutylmalonamide) (II), which is similar to the reported earlier 2,2'-[1,2phenylenebis(methylene) ] bis $\left(N, N, N^{\prime}, N^{\prime}\right.$-tetrabutylmalonamide) (III) (Tyumentsev et al., 2016), from the respective tetraethyl 2,2'-phthaloyldimalonate (IV). In turn (IV) was to be made by the reaction of diethyl malonate with phthaloyl chloride. It is already known that acid chlorides react with diethyl malonate when treated with a combination of triethylamine and a mild Lewis acid (magnesium chloride) in acetonitrile (Rathke \& Cowan, 1985). Instead of (IV), an organic product, which contained two ethyl groups in different electronic environments, was obtained in this reaction. Crystals of this compound were grown and examined with singlecrystal X-ray diffractometry, and the product was found to be the title compound, (I). The formation of (I) can be rationalized by the nucleophilic attack of the oxygen atom (in an enol form) of the keto-diethylmalonate group on the carbon atom of the unreacted acid chloride group. The mechanism of the formation of (I) was proposed by Naik et al. (1988), who obtained this compound by another reaction. 


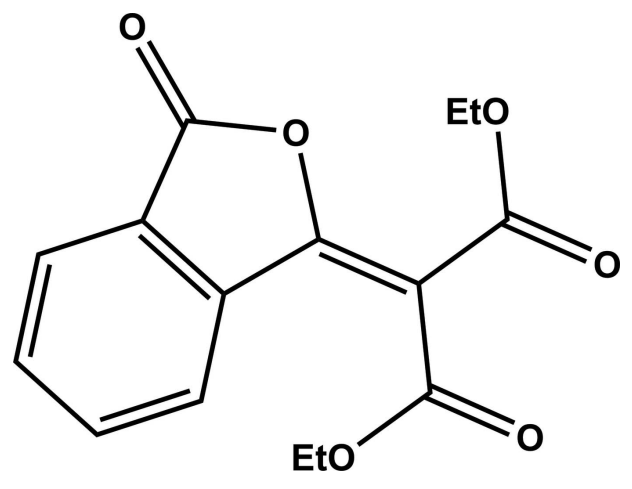

\section{Structural commentary}

Compound (I) crystallizes with one molecule in the asymmetric unit (Fig. 1). Atoms C8, C9, C10, C11, C12, O9 and $\mathrm{O} 10$ are almost coplanar with the isobenzofuran unit (r.m.s. deviation $=0.024 \AA$ ), as shown by the dihedral angle of $5.45(3)^{\circ}$ between these groupings. This mean plane is intercepted nearly perpendicularly by the mean plane of the other ester group (O12, C12, O13, C13, and C14), with a dihedral angle of $83.30(3)^{\circ}$ and the torsion angles $\mathrm{C} 9-\mathrm{C} 8-\mathrm{C} 12-\mathrm{O} 12$ and $\mathrm{C} 9-\mathrm{C} 8-\mathrm{C} 12-\mathrm{O} 13$ of $90.2(1)^{\circ}$ and $-89.6(1)^{\circ}$, respectively. The bond lengths in the carbocyclic ring of the isobenzofuran unit range from 1.386 (2) $\AA$ to 1.398 (2) $\AA$. In the heterocyclic furan ring the distances $\mathrm{C} 2-\mathrm{C} 2 \mathrm{~A}=$ 1.469 (2) $\AA$ and $\mathrm{C} 6 A-\mathrm{C} 7=1.472$ (2) $\AA$ are very similar, while the $\mathrm{C} 2-\mathrm{O} 1$ and $\mathrm{C} 7-\mathrm{O} 1$ distances are significantly different.

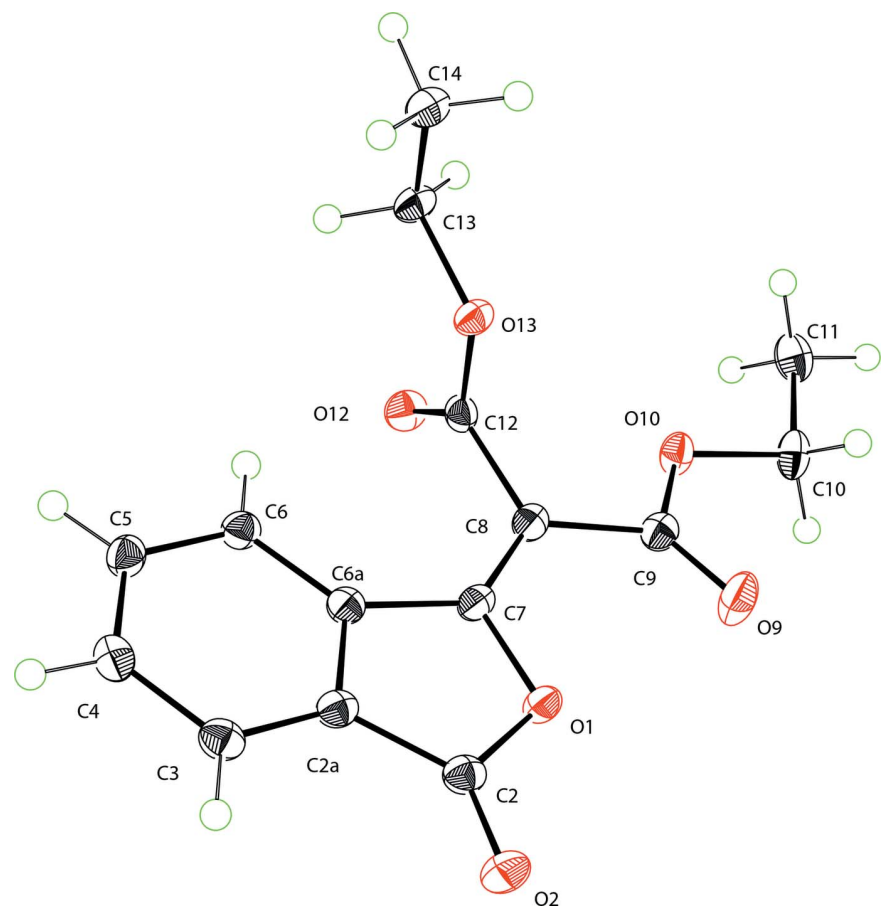

Figure 1

The molecular structure of (I), showing displacement ellipsoids drawn at the $50 \%$ probability level.
Table 1

Hydrogen-bond geometry $\left(\AA{ }^{\circ}\right)$.

\begin{tabular}{lllll}
\hline$D-\mathrm{H} \cdots A$ & $D-\mathrm{H}$ & $\mathrm{H} \cdots A$ & $D \cdots A$ & $D-\mathrm{H} \cdots A$ \\
\hline $\mathrm{C} 5-\mathrm{H} 5 \cdots \mathrm{O} 9^{\mathrm{i}}$ & 0.95 & 2.48 & $3.0689(18)$ & 120 \\
\hline
\end{tabular}

Symmetry code: (i) $x-1, y, z$.

The C2-O1 bond distance of 1.394 (1) A perfectly matches the corresponding distances in phthalic anhydride [1.396 (5) $\AA$ and 1.393 (6) $\AA$ (Bates \& Cutler, 1977)]. The shorter C7-O1 distance of 1.385 (1) $\AA$ strongly suggests that the bond between the endocyclic oxygen atom $\mathrm{O} 1$ and the non-carbonyl carbon atom $\mathrm{C} 7$ has an order greater than 1 . In the diethyl malonate fragment the distances $\mathrm{C} 8-\mathrm{C} 9[1.489$ (2) $\AA]$ and $\mathrm{C} 8-\mathrm{C} 12[1.507(1) \AA]$ are different most likely due to the particular conformation adopted by the molecule. The bond lengths for the atoms, associated with both the furan ring of the isobenzofuran unit and the diethyl malonate fragment, indicate that the dipolar resonance form (Ia) of (I) makes a considerable contribution to its overall molecular electronic structure (Fig. 2).

According to the structure of the resonance form (Ia) a partial positive charge is localized on the oxygen atom of the heterocyclic furan ring, and one of the carbonyl oxygen atoms of the diethyl malonate fragment carries a partial negative charge, which should lead to an electrostatic attraction of these two oxygen atoms. In the structure of (I) the $\mathrm{O} 1$ and $\mathrm{O} 9$ atoms are nearly coplanar (the torsion angles $\mathrm{C} 7-\mathrm{C} 8-\mathrm{C} 9-$ $\mathrm{O} 9$ and $\mathrm{C} 9-\mathrm{C} 8-\mathrm{C} 7-\mathrm{O} 1$ equal to $-10.6(2)^{\circ}$ and $0.7(2)^{\circ}$,

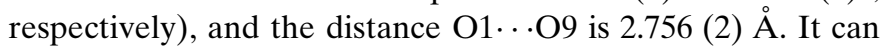<smiles>CCOC(=O)C(C(=O)OCC)=C1OC(=O)c2ccccc21</smiles>

(I)
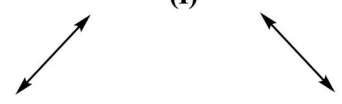<smiles>CCOC(=O)C(C1=[O+]C(=O)c2ccccc21)=C([O-])OCC</smiles>

(Ia)<smiles>CCOC(=O)C(C(=O)OCC)=C1OC(=O)c2ccccc21</smiles>

(Ib)
Figure 2

Chemical diagram of a molecule (I) and its possible resonance forms (Ia) and (Ib). 


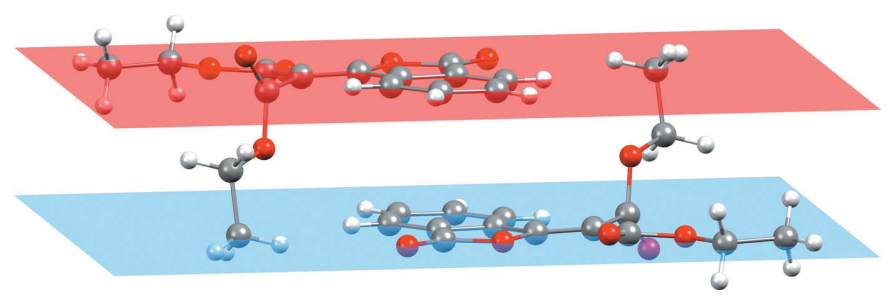

Figure 3

Molecules of (I) interacting via parallel-displaced $\pi-\pi$ stacking.

be argued that simple electrostatic attraction is responsible for this close contact.

\section{Supramolecular features}

The possible non van der Waals contact in the crystal of (I) is a very weak $\mathrm{C}-\mathrm{H} \cdots \mathrm{O}$ interaction (Table 1 ), which links the molecules into [100] $C(8)$ chains. A parallel-displaced $\pi-\pi$ stacking interaction between molecules of (I) is observed with an interplanar distance of $3.423 \AA$ (Fig. 3) and intermolecular furan-benzene and benzene-benzene centroid-to-centroid distances of 3.5379 (13) and 3.7859 (14) Å, respectively.

\section{Database survey}

In the structure of 3-(3-oxo-1,3-dihydroisobenzofuran-1-ylidene)pentane-2,4-dione (HIFQUJ; Portilla et al., 2007) the dominant resonance form resembles (Ib). No contact was observed between the endocyclic oxygen atom and the carbonyl oxygen atom of the acetyl group (the distance between these atoms exceeds $4 \AA$ ). In 2-methoxyethyl 3-oxo2-(3-oxo-2-benzofuran-1(3H)-ylidene)butanoate (UBAVIE; Mkrtchyan et al., 2011), no close contacts exist between the endocyclic oxygen atom and any other oxygen atoms.

In methyl 4,4-dimethyl-3-oxo-2-(3-oxo-2-benzofuran1(3H)-ylidene)pentanoate (UBAVEA; Mkrtchyan et al., 2011), neither of the two carbonyl oxygen atoms of the methyl 4,4-dimethyl-3-oxopentanoate fragment are within the same plane as the isobenzofuran unit. The shortest intermolecular $\mathrm{O}$...O contact is $3.161 \AA$, which occurs between the endocyclic oxygen atom and that carbonyl oxygen atom, which is closest to the plane of the isobenzofuran unit. The torsion angle $\mathrm{O} 4-\mathrm{C} 15-\mathrm{C} 9-\mathrm{C} 8$ in UBAVEA is $26.81^{\circ}$, while the corresponding torsion angle in $(\mathbf{I}), \mathrm{C} 7-\mathrm{C} 8-\mathrm{C} 9-\mathrm{O} 9$, is $10.6(2)^{\circ}$.

\section{Synthesis and crystallization}

The title compound was prepared by the reaction of diethyl malonate with phthaloyl chloride in acetonitrile in the presence of triethylamine and magnesium chloride (Rathke \& Cowan, 1985). The reagents for the synthesis were purchased from Aldrich and were used as supplied. The crude product was washed with petroleum ether on filter paper and recrystallized from cyclohexane solution as colorless crystals $(69 \%$
Table 2

Experimental details.

\begin{tabular}{|c|c|}
\hline \multicolumn{2}{|l|}{ Crystal data } \\
\hline Chemical formula & $\mathrm{C}_{15} \mathrm{H}_{14} \mathrm{O}_{6}$ \\
\hline$M_{\mathrm{r}}$ & 290.27 \\
\hline Crystal system, space group & Triclinic, $P \overline{1}$ \\
\hline Temperature $(\mathrm{K})$ & 93 \\
\hline$a, b, c(\AA)$ & $7.942(2), 9.453(3), 10.226(2)$ \\
\hline$\alpha, \beta, \gamma\left({ }^{\circ}\right)$ & $67.706(15), 72.228(16), 86.41(2)$ \\
\hline$V\left(\AA^{3}\right)$ & $675.2(3)$ \\
\hline$Z$ & 2 \\
\hline Radiation type & Мо $K \alpha$ \\
\hline$\mu\left(\mathrm{mm}^{-1}\right)$ & 0.11 \\
\hline Crystal size $(\mathrm{mm})$ & $0.30 \times 0.20 \times 0.15$ \\
\hline \multicolumn{2}{|l|}{ Data collection } \\
\hline Diffractometer & Rigaku XtaLAB P200 \\
\hline Absorption correction & $\begin{array}{l}\text { Multi-scan (REQAB; Rigaku, } \\
\text { 1998) }\end{array}$ \\
\hline$T_{\min }, T_{\max }$ & $0.829,0.983$ \\
\hline $\begin{array}{l}\text { No. of measured, independent and } \\
\text { observed }\left[F^{2}>2.0 \sigma\left(F^{2}\right)\right] \text { reflec- } \\
\text { tions }\end{array}$ & $9890,2466,2278$ \\
\hline$R_{\mathrm{int}}$ & 0.031 \\
\hline$(\sin \theta / \lambda)_{\max }\left(\AA^{-1}\right)$ & 0.602 \\
\hline \multicolumn{2}{|l|}{ Refinement } \\
\hline$R\left[F^{2}>2 \sigma\left(F^{2}\right)\right], w R\left(F^{2}\right), S$ & $0.028,0.078,1.07$ \\
\hline No. of reflections & 2466 \\
\hline No. of parameters & 192 \\
\hline $\mathrm{H}$-atom treatment & H-atom parameters constrained \\
\hline$\Delta \rho_{\max }, \Delta \rho_{\min }\left(\mathrm{e} \AA^{-3}\right)$ & $0.25,-0.18$ \\
\hline
\end{tabular}

Computer programs: CrystalClear-SM Expert (Rigaku, 2014), SIR2011 (Burla et al., 2012), SHELXL2013 (Sheldrick, 2008) and CrystalStructure (Rigaku, 2014).

yield); m.p. 345-346 K. ${ }^{1} \mathrm{H}$ NMR (400 MHz, $\left.\mathrm{CDCl}_{3}\right) \delta 1.38(\mathrm{~m}$, $6 \mathrm{H}), 4.39(m, 4 \mathrm{H}), 7.72(t, J=6.7 \mathrm{~Hz}, 1 \mathrm{H}), 7.80(t, J=7.4 \mathrm{~Hz}$, $1 \mathrm{H}), 7.99(d, J=7.8 \mathrm{~Hz}, 1 \mathrm{H}), 8.65(d, J=8.2 \mathrm{~Hz}, 1 \mathrm{H}) .{ }^{13} \mathrm{C} \mathrm{NMR}$ $\left(400 \mathrm{MHz}, \mathrm{CDCl}_{3}\right) \delta 135.36 ; 132.93 ; 127.58 ; 126.11 ; 125.84$; 62.18; 62.06; 14.04; 14.01. Found: C, 62.08; $\mathrm{H}, 4.94 \% . \mathrm{C}_{15} \mathrm{H}_{14} \mathrm{O}_{6}$ Theoretical: $\mathrm{C}, 62.07 ; \mathrm{H}, 4.86 \%$. The crystalline product was found to be stable to air, water and brief exposure to $1 \mathrm{M}$ hydrochloric acid.

\section{Refinement}

Crystal data, data collection and structure refinement details are summarized in Table $2 . \mathrm{H}$ atoms were refined using the riding model with $\mathrm{C}-\mathrm{H}=0.95-0.99 \AA$ and $U_{\text {iso }}(\mathrm{H})=$ $1.2 U_{\mathrm{eq}}(\mathrm{C})$.

\section{Funding information}

The research leading to these results received funding from the European Community's Seventh Framework Programme ([FP7/2007-2013]) under grant agreement No. 607411 (MCITN EREAN: European Rare Earth Magnet Recycling Network). This publication reflects only the authors' views, exempting the Community from any liability. Project website: http://www.erean.eu.

\section{References}

Bates, R. B. \& Cutler, R. S. (1977). Acta Cryst. B33, 893-895. 
Burla, M. C., Caliandro, R., Camalli, M., Carrozzini, B., Cascarano, G. L., Giacovazzo, C., Mallamo, M., Mazzone, A., Polidori, G. \& Spagna, R. (2012). J. Appl. Cryst. 45, 357-361.

Mkrtchyan, S., Chilingaryan, Z., Ghazaryan, G., Dede, R., Rasool, N., Rashid, M. A., Villinger, A., Görls, H., Karapetyan, G., Ghochikyan, T. V., Saghiyan, A. \& Langer, P. (2011). Synthesis, pp. 2281-2290.

Naik, S. N., Pandey, B. \& Ayyangar, N. R. (1988). Synth. Commun. 18, 625-632.
Portilla, J., Quiroga, J., Cobo, J., Low, J. N. \& Glidewell, C. (2007). Acta Cryst. C63, o332-o333.

Rathke, M. W. \& Cowan, P. J. (1985). J. Org. Chem. 50, 2622-2624.

Rigaku (1998). REQAB. Rigaku Corporation, Tokyo, Japan.

Rigaku (2014). CrystalClear-SM Expert. Rigaku Corporation, Tokyo, Japan.

Sheldrick, G. M. (2008). Acta Cryst. A64, 112-122.

Tyumentsev, M. S., Foreman, M. R. S. J., Ekberg, C., Matyskin, A. V., Retegan, T. \& Steenari, B.-M. (2016). Hydrometallurgy, 164, 24-30. 


\section{supporting information}

Acta Cryst. (2017). E73, 1576-1579 [https://doi.org/10.1107/S2056989017013962]

\section{Crystal structure and identification of resonance forms of diethyl 2-(3-} oxoiso-1,3-dihydrobenzofuran-1-ylidene)malonate

Mikhail S. Tyumentsev, Mark R. StJ. Foreman, Britt-Marie Steenari and Alexandra M. Z. Slawin

\section{Computing details}

Data collection: CrystalClear-SM Expert (Rigaku, 2014); cell refinement: CrystalClear-SM Expert (Rigaku, 2014); data reduction: CrystalClear-SM Expert (Rigaku, 2014); program(s) used to solve structure: SIR2011 (Burla et al., 2012); program(s) used to refine structure: SHELXL2013 (Sheldrick, 2008); molecular graphics: CrystalStructure (Rigaku, 2014); software used to prepare material for publication: CrystalStructure (Rigaku, 2014).

Diethyl 2-(3-oxo-1,3-dihydro-2-benzofuran-1-ylidene)propanedioate

\section{Crystal data}

$\mathrm{C}_{15} \mathrm{H}_{14} \mathrm{O}_{6}$

$M_{r}=290.27$

Triclinic, $P \overline{1}$

$a=7.942(2) \AA$

$b=9.453(3) \AA$

$c=10.226(2) \AA$

$\alpha=67.706(15)^{\circ}$

$\beta=72.228(16)^{\circ}$

$\gamma=86.41(2)^{\circ}$

$V=675.2(3) \AA^{3}$

\section{Data collection}

Rigaku XtaLAB P200 diffractometer

Detector resolution: 5.814 pixels $\mathrm{mm}^{-1}$ $\omega$ scans

Absorption correction: multi-scan (REQAB; Rigaku, 1998)

$T_{\min }=0.829, T_{\max }=0.983$

9890 measured reflections

\section{Refinement}

Refinement on $F^{2}$

$R\left[F^{2}>2 \sigma\left(F^{2}\right)\right]=0.028$

$w R\left(F^{2}\right)=0.078$

$S=1.07$

2466 reflections 192 parameters 0 restraints

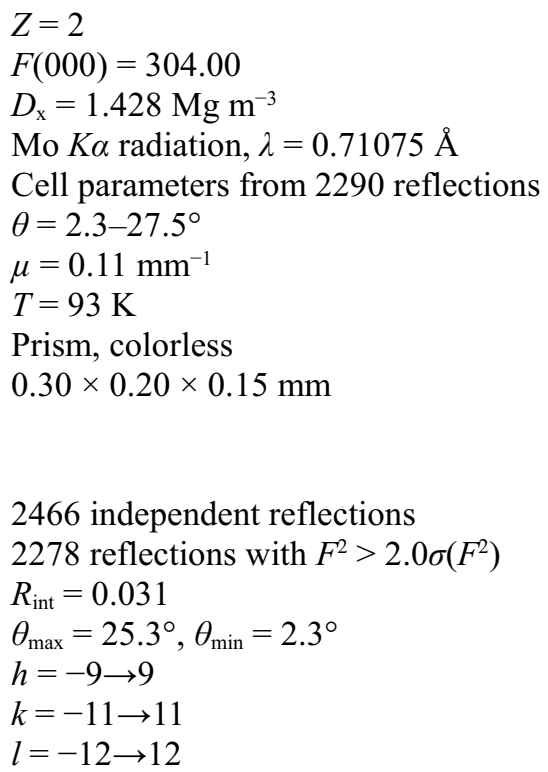

2466 independent reflections 2278 reflections with $F^{2}>2.0 \sigma\left(F^{2}\right)$

$R_{\text {int }}=0.031$

$\theta_{\max }=25.3^{\circ}, \theta_{\min }=2.3^{\circ}$

$h=-9 \rightarrow 9$

$k=-11 \rightarrow 11$

$l=-12 \rightarrow 12$

Primary atom site location: structure-invariant direct methods

Secondary atom site location: difference Fourier map

Hydrogen site location: inferred from neighbouring sites

$\mathrm{H}$-atom parameters constrained 
$w=1 /\left[\sigma^{2}\left(F_{\mathrm{o}}^{2}\right)+(0.0425 P)^{2}+0.0953 P\right]$

where $P=\left(F_{\mathrm{o}}^{2}+2 F_{\mathrm{c}}{ }^{2}\right) / 3$

$(\Delta / \sigma)_{\max }<0.001$
$\Delta \rho_{\max }=0.25 \mathrm{e} \AA^{-3}$
$\Delta \rho_{\min }=-0.18 \mathrm{e} \AA^{-3}$

Special details

Geometry. All esds (except the esd in the dihedral angle between two 1.s. planes) are estimated using the full covariance matrix. The cell esds are taken into account individually in the estimation of esds in distances, angles and torsion angles; correlations between esds in cell parameters are only used when they are defined by crystal symmetry. An approximate (isotropic) treatment of cell esds is used for estimating esds involving 1.s. planes.

Refinement. Refinement was performed using all reflections. The weighted R-factor (wR) and goodness of fit (S) are based on $\mathrm{F}^{2}$. R-factor (gt) are based on $\mathrm{F}$. The threshold expression of $\mathrm{F}^{2}>2.0 \operatorname{sigma}\left(\mathrm{F}^{2}\right)$ is used only for calculating Rfactor (gt).

Fractional atomic coordinates and isotropic or equivalent isotropic displacement parameters $\left(\AA^{2}\right)$

\begin{tabular}{|c|c|c|c|c|}
\hline & $x$ & $y$ & $z$ & $U_{\text {iso }} * / U_{\text {eq }}$ \\
\hline $\mathrm{O} 1$ & $0.78603(9)$ & $0.26223(8)$ & $-0.08336(8)$ & $0.01823(18)$ \\
\hline $\mathrm{O} 2$ & $0.88520(10)$ & $0.18829(9)$ & $-0.27766(9)$ & $0.0250(2)$ \\
\hline O9 & $0.92060(10)$ & $0.33329(10)$ & $0.10424(10)$ & $0.0292(2)$ \\
\hline $\mathrm{O} 10$ & $0.71494(9)$ & $0.40999(9)$ & $0.26677(8)$ & 0.02015 (19) \\
\hline $\mathrm{O} 12$ & $0.34235(10)$ & $0.41829(8)$ & $0.23018(8)$ & 0.02202 (19) \\
\hline $\mathrm{O} 13$ & $0.39408(9)$ & $0.17091(8)$ & $0.34225(8)$ & $0.01784(18)$ \\
\hline $\mathrm{C} 2$ & $0.76082(14)$ & $0.20746(12)$ & $-0.18525(12)$ & $0.0188(2)$ \\
\hline $\mathrm{C} 2 \mathrm{~A}$ & $0.56886(14)$ & $0.18300(12)$ & $-0.15155(12)$ & $0.0176(2)$ \\
\hline $\mathrm{C} 3$ & $0.47681(14)$ & $0.12685(12)$ & $-0.21879(12)$ & $0.0203(2)$ \\
\hline H3 & 0.5367 & 0.0970 & -0.2992 & $0.024 *$ \\
\hline $\mathrm{C} 4$ & $0.29321(14)$ & $0.11623(12)$ & $-0.16343(12)$ & $0.0209(2)$ \\
\hline $\mathrm{H} 4$ & 0.2255 & 0.0764 & -0.2053 & $0.025 *$ \\
\hline $\mathrm{C} 5$ & $0.20674(14)$ & $0.16332(12)$ & $-0.04692(12)$ & $0.0199(2)$ \\
\hline H5 & 0.0809 & 0.1570 & -0.0126 & $0.024 *$ \\
\hline C6 & $0.30017(13)$ & $0.21919(12)$ & $0.02009(12)$ & $0.0181(2)$ \\
\hline H6 & 0.2406 & 0.2513 & 0.0990 & $0.022 *$ \\
\hline C6A & $0.48425(14)$ & $0.22634(11)$ & $-0.03286(11)$ & $0.0164(2)$ \\
\hline $\mathrm{C} 7$ & $0.62379(13)$ & $0.27243(11)$ & $0.01279(11)$ & $0.0164(2)$ \\
\hline $\mathrm{C} 8$ & $0.61249(13)$ & $0.31329(11)$ & $0.12678(12)$ & $0.0170(2)$ \\
\hline C9 & $0.76891(13)$ & $0.35264(12)$ & $0.16046(12)$ & $0.0182(2)$ \\
\hline $\mathrm{C} 10$ & $0.85459(14)$ & $0.45643(13)$ & 0.30998 (13) & $0.0233(3)$ \\
\hline $\mathrm{H} 10 \mathrm{~A}$ & 0.9277 & 0.5452 & 0.2271 & $0.028 *$ \\
\hline H10B & 0.9323 & 0.3712 & 0.3371 & $0.028 *$ \\
\hline C11 & $0.76446(15)$ & $0.49876(14)$ & $0.44073(13)$ & $0.0267(3)$ \\
\hline H11A & 0.6954 & 0.4091 & 0.5228 & $0.032 *$ \\
\hline H11B & 0.6853 & 0.5810 & 0.4131 & $0.032 *$ \\
\hline $\mathrm{H} 11 \mathrm{C}$ & 0.8537 & 0.5338 & 0.4718 & $0.032 *$ \\
\hline C12 & $0.43443(13)$ & $0.31193(12)$ & $0.23574(11)$ & $0.0162(2)$ \\
\hline $\mathrm{C} 13$ & $0.22714(13)$ & $0.15072(12)$ & $0.46014(12)$ & $0.0202(2)$ \\
\hline H13A & 0.1283 & 0.1846 & 0.4175 & $0.024 *$ \\
\hline H13B & 0.2327 & 0.2116 & 0.5192 & $0.024 *$ \\
\hline $\mathrm{C} 14$ & $0.19989(14)$ & $-0.01740(12)$ & $0.55625(12)$ & $0.0220(2)$ \\
\hline $\mathrm{H} 14 \mathrm{~A}$ & 0.1880 & -0.0757 & 0.4978 & $0.026^{*}$ \\
\hline
\end{tabular}




\begin{tabular}{lllll} 
H14B & 0.0921 & -0.0353 & 0.6404 & $0.026^{*}$ \\
H14C & 0.3018 & -0.0506 & 0.5931 & $0.026^{*}$ \\
\hline
\end{tabular}

Atomic displacement parameters $\left(\AA^{2}\right)$

\begin{tabular}{lllllll}
\hline & $U^{11}$ & $U^{22}$ & $U^{33}$ & $U^{12}$ & $U^{13}$ & $U^{23}$ \\
\hline O1 & $0.0135(4)$ & $0.0209(4)$ & $0.0182(4)$ & $0.0008(3)$ & $-0.0019(3)$ & $-0.0076(3)$ \\
O2 & $0.0191(4)$ & $0.0308(5)$ & $0.0227(4)$ & $0.0033(3)$ & $-0.0006(3)$ & $-0.0122(4)$ \\
O9 & $0.0152(4)$ & $0.0410(5)$ & $0.0386(5)$ & $0.0039(3)$ & $-0.0071(4)$ & $-0.0241(4)$ \\
O10 & $0.0160(4)$ & $0.0236(4)$ & $0.0251(4)$ & $0.0010(3)$ & $-0.0078(3)$ & $-0.0125(3)$ \\
O12 & $0.0203(4)$ & $0.0195(4)$ & $0.0236(4)$ & $0.0050(3)$ & $-0.0050(3)$ & $-0.0073(3)$ \\
O13 & $0.0145(4)$ & $0.0173(4)$ & $0.0184(4)$ & $0.0013(3)$ & $-0.0019(3)$ & $-0.0056(3)$ \\
C2 & $0.0194(5)$ & $0.0169(5)$ & $0.0174(5)$ & $0.0022(4)$ & $-0.0042(4)$ & $-0.0048(4)$ \\
C2A & $0.0175(5)$ & $0.0155(5)$ & $0.0167(5)$ & $0.0024(4)$ & $-0.0038(4)$ & $-0.0040(4)$ \\
C3 & $0.0233(6)$ & $0.0199(5)$ & $0.0179(5)$ & $0.0036(4)$ & $-0.0061(4)$ & $-0.0080(4)$ \\
C4 & $0.0226(5)$ & $0.0202(6)$ & $0.0220(6)$ & $0.0018(4)$ & $-0.0103(5)$ & $-0.0075(5)$ \\
C5 & $0.0165(5)$ & $0.0203(5)$ & $0.0215(6)$ & $0.0022(4)$ & $-0.0066(4)$ & $-0.0060(4)$ \\
C6 & $0.0171(5)$ & $0.0188(5)$ & $0.0174(5)$ & $0.0027(4)$ & $-0.0044(4)$ & $-0.0066(4)$ \\
C6A & $0.0174(5)$ & $0.0136(5)$ & $0.0162(5)$ & $0.0015(4)$ & $-0.0055(4)$ & $-0.0033(4)$ \\
C7 & $0.0134(5)$ & $0.0140(5)$ & $0.0176(5)$ & $0.0012(4)$ & $-0.0028(4)$ & $-0.0032(4)$ \\
C8 & $0.0153(5)$ & $0.0146(5)$ & $0.0188(5)$ & $0.0012(4)$ & $-0.0047(4)$ & $-0.0043(4)$ \\
C9 & $0.0173(5)$ & $0.0157(5)$ & $0.0201(5)$ & $0.0007(4)$ & $-0.0051(4)$ & $-0.0055(4)$ \\
C10 & $0.0190(5)$ & $0.0230(6)$ & $0.0338(7)$ & $0.0014(4)$ & $-0.0136(5)$ & $-0.0126(5)$ \\
C11 & $0.0286(6)$ & $0.0274(6)$ & $0.0314(7)$ & $0.0030(5)$ & $-0.0162(5)$ & $-0.0137(5)$ \\
C12 & $0.0164(5)$ & $0.0176(5)$ & $0.0169(5)$ & $0.0001(4)$ & $-0.0071(4)$ & $-0.0073(4)$ \\
C13 & $0.0153(5)$ & $0.0220(6)$ & $0.0198(6)$ & $0.0009(4)$ & $-0.0003(4)$ & $-0.0081(5)$ \\
C14 & $0.0188(5)$ & $0.0213(6)$ & $0.0217(6)$ & $-0.0005(4)$ & $-0.0023(4)$ & $-0.0064(5)$ \\
& & & & & &
\end{tabular}

Geometric parameters $\left(A,{ }^{\circ}\right)$

\begin{tabular}{llll}
\hline $\mathrm{O} 1-\mathrm{C} 7$ & $1.3854(12)$ & $\mathrm{C} 6-\mathrm{C} 6 \mathrm{~A}$ & $1.3916(15)$ \\
$\mathrm{O} 1-\mathrm{C} 2$ & $1.3940(13)$ & $\mathrm{C} 6-\mathrm{H} 6$ & 0.9500 \\
$\mathrm{O} 2-\mathrm{C} 2$ & $1.1997(13)$ & $\mathrm{C} 6 \mathrm{~A}-\mathrm{C} 7$ & $1.4719(14)$ \\
$\mathrm{O} 9-\mathrm{C} 9$ & $1.2019(13)$ & $\mathrm{C} 7-\mathrm{C} 8$ & $1.3377(15)$ \\
$\mathrm{O} 10-\mathrm{C} 9$ & $1.3391(13)$ & $\mathrm{C} 8-\mathrm{C} 9$ & $1.4888(14)$ \\
$\mathrm{O} 10-\mathrm{C} 10$ & $1.4579(13)$ & $\mathrm{C} 8-\mathrm{C} 12$ & $1.5070(14)$ \\
$\mathrm{O} 12-\mathrm{C} 12$ & $1.1995(13)$ & $\mathrm{C} 10-\mathrm{C} 11$ & $1.4986(16)$ \\
$\mathrm{O} 13-\mathrm{C} 12$ & $1.3435(13)$ & $\mathrm{C} 10-\mathrm{H} 10 \mathrm{~A}$ & 0.9900 \\
$\mathrm{O} 13-\mathrm{C} 13$ & $1.4591(12)$ & $\mathrm{C} 10-\mathrm{H} 10 \mathrm{~B}$ & 0.9900 \\
$\mathrm{C} 2-\mathrm{C} 2 \mathrm{~A}$ & $1.4695(15)$ & $\mathrm{C} 11-\mathrm{H} 11 \mathrm{~A}$ & 0.9800 \\
$\mathrm{C} 2 \mathrm{~A}-\mathrm{C} 3$ & $1.3862(15)$ & $\mathrm{C} 11-\mathrm{H} 11 \mathrm{~B}$ & 0.9800 \\
$\mathrm{C} 2 \mathrm{~A}-\mathrm{C} 6 \mathrm{~A}$ & $1.3919(15)$ & $\mathrm{C} 11-\mathrm{H} 11 \mathrm{C}$ & 0.9800 \\
$\mathrm{C} 3-\mathrm{C} 4$ & $1.3886(16)$ & $\mathrm{C} 13-\mathrm{C} 14$ & $1.5055(16)$ \\
$\mathrm{C} 3-\mathrm{H} 3$ & 0.9500 & $\mathrm{C} 13-\mathrm{H} 13 \mathrm{~A}$ & 0.9900 \\
$\mathrm{C} 4-\mathrm{C} 5$ & $1.3978(16)$ & $\mathrm{C} 13-\mathrm{H} 13 \mathrm{~B}$ & 0.9900 \\
$\mathrm{C} 4-\mathrm{H} 4$ & 0.9500 & $\mathrm{C} 14-\mathrm{H} 14 \mathrm{~A}$ & 0.9800 \\
$\mathrm{C} 5-\mathrm{C} 6$ & $1.3904(15)$ & $\mathrm{C} 14-\mathrm{H} 14 \mathrm{~B}$ & 0.9800 \\
$\mathrm{C} 5-\mathrm{H} 5$ & 0.9500 & $\mathrm{C} 14-\mathrm{H} 14 \mathrm{C}$ & 0.9800
\end{tabular}




\begin{tabular}{|c|c|c|c|}
\hline $\mathrm{C} 7-\mathrm{O} 1-\mathrm{C} 2$ & $109.90(8)$ & $\mathrm{O} 9-\mathrm{C} 9-\mathrm{O} 10$ & $124.58(10)$ \\
\hline $\mathrm{C} 9-\mathrm{O} 10-\mathrm{C} 10$ & $115.85(8)$ & $\mathrm{O} 9-\mathrm{C} 9-\mathrm{C} 8$ & $125.85(10)$ \\
\hline $\mathrm{C} 12-\mathrm{O} 13-\mathrm{C} 13$ & $116.01(8)$ & $\mathrm{O} 10-\mathrm{C} 9-\mathrm{C} 8$ & $109.55(9)$ \\
\hline $\mathrm{O} 2-\mathrm{C} 2-\mathrm{O} 1$ & $120.62(10)$ & $\mathrm{O} 10-\mathrm{C} 10-\mathrm{C} 11$ & $106.67(9)$ \\
\hline $\mathrm{O} 2-\mathrm{C} 2-\mathrm{C} 2 \mathrm{~A}$ & $132.18(11)$ & $\mathrm{O} 10-\mathrm{C} 10-\mathrm{H} 10 \mathrm{~A}$ & 110.4 \\
\hline $\mathrm{O} 1-\mathrm{C} 2-\mathrm{C} 2 \mathrm{~A}$ & $107.20(9)$ & $\mathrm{C} 11-\mathrm{C} 10-\mathrm{H} 10 \mathrm{~A}$ & 110.4 \\
\hline $\mathrm{C} 3-\mathrm{C} 2 \mathrm{~A}-\mathrm{C} 6 \mathrm{~A}$ & $122.56(10)$ & $\mathrm{O} 10-\mathrm{C} 10-\mathrm{H} 10 \mathrm{~B}$ & 110.4 \\
\hline $\mathrm{C} 3-\mathrm{C} 2 \mathrm{~A}-\mathrm{C} 2$ & $129.50(10)$ & $\mathrm{C} 11-\mathrm{C} 10-\mathrm{H} 10 \mathrm{~B}$ & 110.4 \\
\hline $\mathrm{C} 6 \mathrm{~A}-\mathrm{C} 2 \mathrm{~A}-\mathrm{C} 2$ & $107.95(9)$ & $\mathrm{H} 10 \mathrm{~A}-\mathrm{C} 10-\mathrm{H} 10 \mathrm{~B}$ & 108.6 \\
\hline $\mathrm{C} 2 \mathrm{~A}-\mathrm{C} 3-\mathrm{C} 4$ & $117.04(10)$ & $\mathrm{C} 10-\mathrm{C} 11-\mathrm{H} 11 \mathrm{~A}$ & 109.5 \\
\hline $\mathrm{C} 2 \mathrm{~A}-\mathrm{C} 3-\mathrm{H} 3$ & 121.5 & $\mathrm{C} 10-\mathrm{C} 11-\mathrm{H} 11 \mathrm{~B}$ & 109.5 \\
\hline $\mathrm{C} 4-\mathrm{C} 3-\mathrm{H} 3$ & 121.5 & $\mathrm{H} 11 \mathrm{~A}-\mathrm{C} 11-\mathrm{H} 11 \mathrm{~B}$ & 109.5 \\
\hline $\mathrm{C} 3-\mathrm{C} 4-\mathrm{C} 5$ & $120.88(10)$ & $\mathrm{C} 10-\mathrm{C} 11-\mathrm{H} 11 \mathrm{C}$ & 109.5 \\
\hline $\mathrm{C} 3-\mathrm{C} 4-\mathrm{H} 4$ & 119.6 & $\mathrm{H} 11 \mathrm{~A}-\mathrm{C} 11-\mathrm{H} 11 \mathrm{C}$ & 109.5 \\
\hline $\mathrm{C} 5-\mathrm{C} 4-\mathrm{H} 4$ & 119.6 & $\mathrm{H} 11 \mathrm{~B}-\mathrm{C} 11-\mathrm{H} 11 \mathrm{C}$ & 109.5 \\
\hline $\mathrm{C} 6-\mathrm{C} 5-\mathrm{C} 4$ & $121.67(10)$ & $\mathrm{O} 12-\mathrm{C} 12-\mathrm{O} 13$ & $124.83(10)$ \\
\hline $\mathrm{C} 6-\mathrm{C} 5-\mathrm{H} 5$ & 119.2 & $\mathrm{O} 12-\mathrm{C} 12-\mathrm{C} 8$ & $126.32(10)$ \\
\hline $\mathrm{C} 4-\mathrm{C} 5-\mathrm{H} 5$ & 119.2 & $\mathrm{O} 13-\mathrm{C} 12-\mathrm{C} 8$ & $108.85(8)$ \\
\hline $\mathrm{C} 5-\mathrm{C} 6-\mathrm{C} 6 \mathrm{~A}$ & $117.49(10)$ & $\mathrm{O} 13-\mathrm{C} 13-\mathrm{C} 14$ & $106.78(8)$ \\
\hline $\mathrm{C} 5-\mathrm{C} 6-\mathrm{H} 6$ & 121.3 & $\mathrm{O} 13-\mathrm{C} 13-\mathrm{H} 13 \mathrm{~A}$ & 110.4 \\
\hline $\mathrm{C} 6 \mathrm{~A}-\mathrm{C} 6-\mathrm{H} 6$ & 121.3 & $\mathrm{C} 14-\mathrm{C} 13-\mathrm{H} 13 \mathrm{~A}$ & 110.4 \\
\hline $\mathrm{C} 6-\mathrm{C} 6 \mathrm{~A}-\mathrm{C} 2 \mathrm{~A}$ & $120.32(10)$ & $\mathrm{O} 13-\mathrm{C} 13-\mathrm{H} 13 \mathrm{~B}$ & 110.4 \\
\hline $\mathrm{C} 6-\mathrm{C} 6 \mathrm{~A}-\mathrm{C} 7$ & $132.70(10)$ & $\mathrm{C} 14-\mathrm{C} 13-\mathrm{H} 13 \mathrm{~B}$ & 110.4 \\
\hline $\mathrm{C} 2 \mathrm{~A}-\mathrm{C} 6 \mathrm{~A}-\mathrm{C} 7$ & $106.98(9)$ & $\mathrm{H} 13 \mathrm{~A}-\mathrm{C} 13-\mathrm{H} 13 \mathrm{~B}$ & 108.6 \\
\hline $\mathrm{C} 8-\mathrm{C} 7-\mathrm{O} 1$ & $121.51(9)$ & $\mathrm{C} 13-\mathrm{C} 14-\mathrm{H} 14 \mathrm{~A}$ & 109.5 \\
\hline $\mathrm{C} 8-\mathrm{C} 7-\mathrm{C} 6 \mathrm{~A}$ & $130.55(10)$ & $\mathrm{C} 13-\mathrm{C} 14-\mathrm{H} 14 \mathrm{~B}$ & 109.5 \\
\hline $\mathrm{O} 1-\mathrm{C} 7-\mathrm{C} 6 \mathrm{~A}$ & $107.90(9)$ & $\mathrm{H} 14 \mathrm{~A}-\mathrm{C} 14-\mathrm{H} 14 \mathrm{~B}$ & 109.5 \\
\hline $\mathrm{C} 7-\mathrm{C} 8-\mathrm{C} 9$ & $123.84(9)$ & $\mathrm{C} 13-\mathrm{C} 14-\mathrm{H} 14 \mathrm{C}$ & 109.5 \\
\hline $\mathrm{C} 7-\mathrm{C} 8-\mathrm{C} 12$ & $120.08(9)$ & $\mathrm{H} 14 \mathrm{~A}-\mathrm{C} 14-\mathrm{H} 14 \mathrm{C}$ & 109.5 \\
\hline $\mathrm{C} 9-\mathrm{C} 8-\mathrm{C} 12$ & $115.97(9)$ & $\mathrm{H} 14 \mathrm{~B}-\mathrm{C} 14-\mathrm{H} 14 \mathrm{C}$ & 109.5 \\
\hline $\mathrm{C} 7-\mathrm{O} 1-\mathrm{C} 2-\mathrm{O} 2$ & $-179.09(9)$ & $\mathrm{C} 6-\mathrm{C} 6 \mathrm{~A}-\mathrm{C} 7-\mathrm{O} 1$ & $-177.86(10)$ \\
\hline $\mathrm{C} 7-\mathrm{O} 1-\mathrm{C} 2-\mathrm{C} 2 \mathrm{~A}$ & $0.60(11)$ & $\mathrm{C} 2 \mathrm{~A}-\mathrm{C} 6 \mathrm{~A}-\mathrm{C} 7-\mathrm{O} 1$ & $2.72(11)$ \\
\hline $\mathrm{O} 2-\mathrm{C} 2-\mathrm{C} 2 \mathrm{~A}-\mathrm{C} 3$ & $1.1(2)$ & $\mathrm{O} 1-\mathrm{C} 7-\mathrm{C} 8-\mathrm{C} 9$ & $0.65(16)$ \\
\hline $\mathrm{O} 1-\mathrm{C} 2-\mathrm{C} 2 \mathrm{~A}-\mathrm{C} 3$ & $-178.50(10)$ & $\mathrm{C} 6 \mathrm{~A}-\mathrm{C} 7-\mathrm{C} 8-\mathrm{C} 9$ & $178.04(9)$ \\
\hline $\mathrm{O} 2-\mathrm{C} 2-\mathrm{C} 2 \mathrm{~A}-\mathrm{C} 6 \mathrm{~A}$ & $-179.21(11)$ & $\mathrm{O} 1-\mathrm{C} 7-\mathrm{C} 8-\mathrm{C} 12$ & $-175.47(8)$ \\
\hline $\mathrm{O} 1-\mathrm{C} 2-\mathrm{C} 2 \mathrm{~A}-\mathrm{C} 6 \mathrm{~A}$ & $1.15(11)$ & $\mathrm{C} 6 \mathrm{~A}-\mathrm{C} 7-\mathrm{C} 8-\mathrm{C} 12$ & $1.92(17)$ \\
\hline $\mathrm{C} 6 \mathrm{~A}-\mathrm{C} 2 \mathrm{~A}-\mathrm{C} 3-\mathrm{C} 4$ & $0.54(15)$ & $\mathrm{C} 10-\mathrm{O} 10-\mathrm{C} 9-\mathrm{O} 9$ & $2.55(15)$ \\
\hline $\mathrm{C} 2-\mathrm{C} 2 \mathrm{~A}-\mathrm{C} 3-\mathrm{C} 4$ & $-179.85(10)$ & $\mathrm{C} 10-\mathrm{O} 10-\mathrm{C} 9-\mathrm{C} 8$ & $-179.01(8)$ \\
\hline $\mathrm{C} 2 \mathrm{~A}-\mathrm{C} 3-\mathrm{C} 4-\mathrm{C} 5$ & $1.21(15)$ & $\mathrm{C} 7-\mathrm{C} 8-\mathrm{C} 9-\mathrm{O} 9$ & $-10.62(17)$ \\
\hline $\mathrm{C} 3-\mathrm{C} 4-\mathrm{C} 5-\mathrm{C} 6$ & $-1.41(16)$ & $\mathrm{C} 12-\mathrm{C} 8-\mathrm{C} 9-\mathrm{O} 9$ & $165.65(10)$ \\
\hline $\mathrm{C} 4-\mathrm{C} 5-\mathrm{C} 6-\mathrm{C} 6 \mathrm{~A}$ & $-0.18(15)$ & $\mathrm{C} 7-\mathrm{C} 8-\mathrm{C} 9-\mathrm{O} 10$ & $170.96(9)$ \\
\hline $\mathrm{C} 5-\mathrm{C} 6-\mathrm{C} 6 \mathrm{~A}-\mathrm{C} 2 \mathrm{~A}$ & $1.90(15)$ & $\mathrm{C} 12-\mathrm{C} 8-\mathrm{C} 9-\mathrm{O} 10$ & $-12.77(12)$ \\
\hline $\mathrm{C} 5-\mathrm{C} 6-\mathrm{C} 6 \mathrm{~A}-\mathrm{C} 7$ & $-177.45(10)$ & $\mathrm{C} 9-\mathrm{O} 10-\mathrm{C} 10-\mathrm{C} 11$ & $-173.45(9)$ \\
\hline $\mathrm{C} 3-\mathrm{C} 2 \mathrm{~A}-\mathrm{C} 6 \mathrm{~A}-\mathrm{C} 6$ & $-2.15(15)$ & $\mathrm{C} 13-\mathrm{O} 13-\mathrm{C} 12-\mathrm{O} 12$ & $-1.65(14)$ \\
\hline $\mathrm{C} 2-\mathrm{C} 2 \mathrm{~A}-\mathrm{C} 6 \mathrm{~A}-\mathrm{C} 6$ & $178.17(9)$ & $\mathrm{C} 13-\mathrm{O} 13-\mathrm{C} 12-\mathrm{C} 8$ & $178.17(8)$ \\
\hline $\mathrm{C} 3-\mathrm{C} 2 \mathrm{~A}-\mathrm{C} 6 \mathrm{~A}-\mathrm{C} 7$ & $177.35(9)$ & $\mathrm{C} 7-\mathrm{C} 8-\mathrm{C} 12-\mathrm{O} 12$ & $-93.36(14)$ \\
\hline
\end{tabular}


supporting information

$\mathrm{C} 2-\mathrm{C} 2 \mathrm{~A}-\mathrm{C} 6 \mathrm{~A}-\mathrm{C} 7$
$\mathrm{C} 2-\mathrm{O} 1-\mathrm{C} 7-\mathrm{C} 8$
$\mathrm{C} 2-\mathrm{O} 1-\mathrm{C} 7-\mathrm{C} 6 \mathrm{~A}$
$\mathrm{C} 6-\mathrm{C} 6 \mathrm{~A}-\mathrm{C} 7-\mathrm{C} 8$
$\mathrm{C} 2 \mathrm{~A}-\mathrm{C} 6 \mathrm{~A}-\mathrm{C} 7-\mathrm{C} 8$

$-2.32(11)$

$175.88(9)$

$-2.03(10)$

4.48 (19)

$-174.94(11)$

$\begin{array}{ll}\mathrm{C} 9-\mathrm{C} 8-\mathrm{C} 12-\mathrm{O} 12 & 90.22(13) \\ \mathrm{C} 7-\mathrm{C} 8-\mathrm{C} 12-\mathrm{O} 13 & 86.82(11) \\ \mathrm{C} 9-\mathrm{C} 8-\mathrm{C} 12-\mathrm{O} 13 & -89.60(10) \\ \mathrm{C} 12-\mathrm{O} 13-\mathrm{C} 13-\mathrm{C} 14 & 173.90(8)\end{array}$

C9- 8 - $-\mathrm{C} 12-\mathrm{O} 12$

$90.22(13)$

$-89.60(10)$

$\mathrm{C} 9-\mathrm{C} 8-\mathrm{C} 12-\mathrm{O} 13$

$173.90(8)$

Hydrogen-bond geometry $\left(A,{ }^{\circ}\right)$

\begin{tabular}{lllll}
\hline$D-\mathrm{H} \cdots A$ & $D-\mathrm{H}$ & $\mathrm{H} \cdots A$ & $D \cdots A$ & $D-\mathrm{H} \cdots A$ \\
\hline $\mathrm{C} 5-\mathrm{H} 5 \cdots \mathrm{O}^{\mathrm{i}}$ & 0.95 & 2.48 & $3.0689(18)$ & 120 \\
\hline
\end{tabular}

Symmetry code: (i) $x-1, y, z$. 